\title{
Lower Bounds from Fitness Levels Made Easy
}

\author{
Benjamin Doerr \\ Laboratoire d'Informatique (LIX) \\ CNRS \\ École Polytechnique \\ Institut Polytechnique de Paris \\ Palaiseau, France
}

\author{
Timo Kötzing \\ Hasso Plattner Institute \\ Potsdam, Germany
}

\begin{abstract}
One of the first and easy to use techniques for proving run time bounds for evolutionary algorithms is the so-called method of fitness levels by Wegener. It uses a partition of the search space into a sequence of levels which are traversed by the algorithm in increasing order, possibly skipping levels. An easy, but often strong upper bound for the run time can then be derived by adding the reciprocals of the probabilities to leave the levels (or upper bounds for these). Unfortunately, a similarly effective method for proving lower bounds has not yet been established. The strongest such method, proposed by Sudholt (2013), requires a careful choice of the viscosity parameters $\gamma_{i, j}, 0 \leq i<j \leq n$.

In this paper we present two new variants of the method, one for upper and one for lower bounds. Besides the level leaving probabilities, they only rely on the probabilities that levels are visited at all. We show that these can be computed or estimated without greater difficulties and apply our method to reprove the following known results in an easy and natural way. (i) The precise run time of the $(1+1)$ EA on LEAdingOnes. (ii) A lower bound for the run time of the $(1+1)$ EA on OneMAX, tight apart from an $O(n)$ term. (iii) A lower bound for the run time of the $(1+1)$ EA on long $k$-paths.
\end{abstract}

\section{CCS CONCEPTS}

- Theory of computation $\rightarrow$ Theory of randomized search heuristics.

\section{KEYWORDS}

Fitness level method, lower bounds, run time analysis

\section{ACM Reference Format:}

Benjamin Doerr and Timo Kötzing. 2021. Lower Bounds from Fitness Levels Made Easy. In 2021 Genetic and Evolutionary Computation Conference (GECCO '21), fuly 10-14, 2021, Lille, France. ACM, New York, NY, USA, 9 pages. https://doi.org/10.1145/3449639.3459352

\section{INTRODUCTION}

The theory of evolutionary computation aims at explaining the behavior of evolutionary algorithms, for example by giving detailed

Permission to make digital or hard copies of all or part of this work for personal or classroom use is granted without fee provided that copies are not made or distributed for profit or commercial advantage and that copies bear this notice and the full citation on the first page. Copyrights for components of this work owned by others than the author(s) must be honored. Abstracting with credit is permitted. To copy otherwise, or republish, to post on servers or to redistribute to lists, requires prior specific permission and/or a fee. Request permissions from permissions@acm.org.

GECCO '21, fuly 10-14, 2021, Lille, France

(C) 2021 Copyright held by the owner/author(s). Publication rights licensed to ACM ACM ISBN 978-1-4503-8350-9/21/07 . \$ \$15.00

https://doi.org/10.1145/3449639.3459352 run time analyses of such algorithms on certain test functions, defined on some search space (for this paper we will focus on $\left.\{0,1\}^{n}\right)$. The first general method for conducting such analyzes is the fitness level method (FLM) [38, 39]. The idea of this method is as follows. We partition the search space into a number $m$ of sections ("levels") in a linear fashion, so that all elements of later levels have better fitness than all elements of earlier levels. For the algorithm to be analyzed we regard the best-so-far individual and the level it is in. Since the best-so-far individual can never move to lower levels, it will visit each level at most once (possibly staying there for some time). Suppose we can show that, for any level $i<m$ which the algorithm is currently in, the probability to leave this level is at least $p_{i}$. Then, bounding the expected waiting for leaving a level $i$ by $1 / p_{i}$, we can derive an upper bound for the run time of $\sum_{i=1}^{m-1} 1 / p_{i}$ by pessimistically assuming that we visit (and thus have to leave) each level $i<m$ before reaching the target level $m$. The fitness level method allows for simple and intuitive proofs and has therefore frequently been applied. Variations of it come with tail bounds [41], work for parallel EAs [28], or admit non-elitist EAs $[4,5,14,29]$.

While very effective for proving upper bounds, it seems much harder to use fitness level arguments to prove lower bounds (see Theorem 3.5 for an early attempt). The first (and so far only) to devise a fitness level-based lower bound method that gives competitive bounds was Sudholt [37]. His approach uses viscosity parameters $\gamma_{i, j}, 0 \leq i<j \leq n$, which control the probability of the algorithm to jump from one level $i$ to a higher level $j$ (see Section 3.3 for details). While this allows for deriving strong results, the application is rather technical due to the many parameters and the restrictions they have to fulfill.

In this paper, we propose a new variant of the FLM for lower bounds, which is easier to use and which appears more intuitive. For each level $i$, we regard the visit probability $v_{i}$, that is, the probability that level $i$ is visited at all during a run of the algorithm. This way we can directly characterize the run time of the algorithm as $\sum_{i=1}^{m-1} v_{i} / p_{i}$ when $p_{i}$ is the precise probability to leave level $i$ independent of where on level $i$ the algorithm is. When only estimates for these quantities are known, e.g., because the level leaving probability is not independent from the current state, then we obtain the corresponding upper or lower bounds on the expected run time (see Section 3.4 for details).

We first use this method to give the precise expected run time of the $(1+1)$ EA on LEADINGOnes in Section 4. While this run time was already well-understood before, it serves as a simple demonstration of the ease with which our method can be applied.

Next, in Section 5, we give a bound on the expected run time of the $(1+1)$ EA on ONEMAX, precise apart from terms of order $\Theta(n)$. 
Such bounds have also been known before, but needed much deeper methods (see Section 5.3 for a detailed discussion). Sudholt's lower bound method has also been applied to this problem, but gave a slightly weaker bound deviating from the truth by an $O(n \log \log n)$ term. In addition to the precise result, we feel that our FLM with visit probabilities gives a clearer structure of the proof than the previous works.

Finally, in Section 6, we consider the $(1+1)$ EA on so-called long $k$-paths. We show how the FLM with visit probabilities can give results comparable to those of the FLM with viscosities while again being much simpler to apply.

\section{THE $(1+1)$ EA}

In this paper we consider exactly one randomized search heuristic, the $(1+1)$ EA. It maintains a single individual, the best it has seen so far. Each iteration it uses standard bit mutation with mutation rate $p \in(0,1)$ (flipping each bit of the bit string independently with probability $p$ ) and keeps the result if and only if it is at least as good as the current individual under a given fitness function $f$. We give a more formal definition in Algorithm 1.

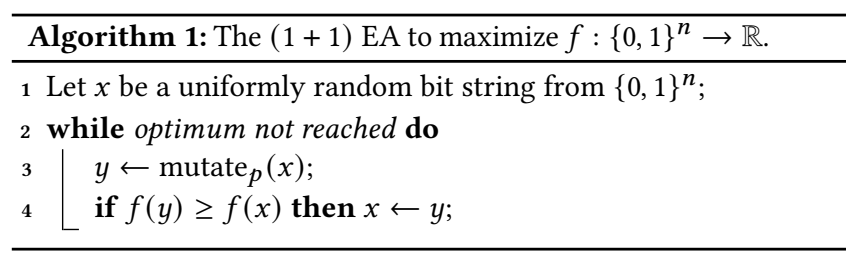

\section{THE FITNESS LEVEL METHODS}

The fitness level method is typically phrased in terms of a fitnessbased partition, that is, a partition of the search space into sets $A_{1}, \ldots, A_{m}$ such that elements of later sets have higher fitness. We first introduce this concept and abstract away from it to ease the notation. After this, in Section 3.2, we state the original FLM. In Section 3.3 we describe the lower bound based on the FLM from Sudholt [37], before presenting our own variant, the FLM with visit probabilities, in Section 3.4.

\subsection{Level Processes}

Definition 3.1 (Fitness-Based Partition [39]). Let $f:\{0,1\}^{n} \rightarrow \mathbb{R}$ be a fitness function. A partition $A_{1}, \ldots, A_{m}$ of $\{0,1\}^{n}$ is called a fitness-based partition if for all $i, j \leq m$ with $i<j$ and $x \in A_{i}$, $y \in A_{j}$, we have $f(x)<f(y)$.

We will use the shorthands $A_{\geq i}=\bigcup_{j=i}^{m} A_{j}$ and $A_{\leq i}=\bigcup_{j=1}^{i} A_{j}$. In order to simplify our notation, we focus on processes on [1..m] (the levels) with underlying Markov chain as follows.

Definition 3.2 (Non-decreasing Level Process). A stochastic process $\left(X_{t}\right)_{t}$ on $[1 . . m]$ is called a non-decreasing level process if and only if (i) there exists a Markov process $\left(Y_{t}\right)_{t}$ over a state space $S$ such that there is an $\ell: S \rightarrow[1 . . m]$ with $\ell\left(Y_{t}\right)=X_{t}$ for all $t$, and (ii) the process $\left(X_{t}\right)_{t}$ is non-decreasing, that is, we have $X_{t+1} \geq X_{t}$ with probability one for all $t$.
We later want to analyze algorithms in terms of non-decreasing level processes, making the transition as follows. Suppose we have an algorithm with state space $\{0,1\}^{n}$. Denoting by $Y_{t}$ the best among the first $t$ search points generated by the algorithm, this defines a Markov Chain $\left(Y_{t}\right)_{t}$ in the state space $S=\{0,1\}^{n}$, the run of the algorithm. Further, suppose the algorithm optimizes a fitness function $f$ such that the state of the algorithm is non-decreasing in terms of fitness. In order to get a non-decreasing level process, we can now define any fitness-based partition and get a corresponding level function $\ell: S \rightarrow[1 . . m]$ by mapping any $x \in S$ to the unique $i$ with $x \in A_{i}$. Then the process $\left(\ell\left(Y_{t}\right)\right)_{t}$ is a non-decreasing level process.

The main reason for us to use the formal notion of a level process is the property formalized in the following lemma. Essentially, if a level process makes progress with probability at least $p$ in each iteration (regardless of the precise current state), then the expected number of iterations until the process progresses is at most $1 / p$. This situation resembles a geometric distribution, but does not assume independence of the different iterations (one could show that the time to progress is stochastically dominated by a geometric distribution with success rate $p$, but we do not need this level of detail).

Lemma 3.3. Let $\left(X_{t}\right)_{t}$ be a non-decreasing level process with underlying Markov chain $\left(Y_{t}\right)_{t}$ and level function $\ell$. Assume $X_{t}$ starts on some particular level. Let $p \in(0,1]$ be a lower bound on the probability for level process to leave this level regardless of the state of the underlying Markov chain. Then the expected first time $t$ such that $X_{t}$ changes is at most $1 / p$.

Analogously, if $p$ is an upper bound, the expected time $t$ such that $X_{t}$ changes is at least $1 / p$.

Proof. We let $\left(Z_{t}\right)_{t}$ be the stochastic process on $\{0,1\}$ such that $Z_{t}$ is 1 if and only if $X_{t}>X_{0}$. According to our assumptions, we have, for all $t$ before the first time that $Z_{t}=1$, that $E\left[Z_{t+1}-Z_{t} \mid\right.$ $\left.Z_{t}\right] \geq p$. From the additive drift theorem $[22,31]$ we obtain that the expected first time such that $Z_{t}=1$ is bounded by $1 / p$ as desired. The "analogously" clause follows analogously.

\subsection{Original Fitness Level Method}

The following theorem contains the original Fitness Level Method and makes the basic principle formal.

Theorem 3.4 (Fitness Level Method, Upper Bound [39]). Let $\left(X_{t}\right)_{t}$ be a non-decreasing level process (as detailed in Definition 3.2).

For all $i \in[1 . . m-1]$, let $p_{i}$ be a lower bound on the probability of a state change of $\left(X_{t}\right)_{t}$, conditional on being in state $i$. Then the expected time for $\left(X_{t}\right)_{t}$ to reach the state $m$ is

$$
E[T] \leq \sum_{i=1}^{m-1} \frac{1}{p_{i}} .
$$

This bound is very simple, yet strong. It is based on the idea that, in the worst case, all levels have to be visited sequentially. Note that one can improve this bound (slightly) by considering only those levels which come after the (random) start level $X_{0}$ (by changing the start of the sum to $X_{0}$ instead of 1 ). Intuitively, low levels that are never visited do not need to be left. 
There is a lower bound based on the observation that at least the initial level has to be left (if it was not the last level).

Theorem 3.5 (Fitness Level Method, LOWer bound [39]). Let $\left(X_{t}\right)_{t}$ be a non-decreasing level process (as detailed in Definition 3.2).

For all $i \in[1 . . m-1]$, let $p_{i}$ be an upper bound on the probability of a state change, conditional on being in state $i$. Then the expected time for $\left(X_{t}\right)_{t}$ to reach the state $m$ is

$$
E[T] \geq \sum_{i=0}^{m-1} \operatorname{Pr}\left[X_{0}=i\right] \frac{1}{p_{i}} .
$$

This bound is very weak since it assumes that the first improvement on the initial search point already finds the optimum.

We note, very briefly, that a second main analysis method, drift analysis, also has additional difficulties with lower bounds. Additive drift [22], multiplicative drift [13], and variable drift [27, 32] all easily give upper bounds for run times, however, only the additive drift theorem yields lower bounds with the same ease. The existing multiplicative $[8,16,40]$ and variable $[9,11,19,21]$ drift theorems for lower bounds all need significantly stronger assumptions than their counterparts for upper bounds.

\subsection{Fitness Level Method with Viscosity}

While the upper bound above is strong and useful, the lower bound is typically not strong enough to give more than a trivial bound. Sudholt [37] gave a refinement of the method by considering bounds on the transition probabilities from one level to another.

Theorem 3.6 (Fitness LeVel Method with Viscosity, Lower BOUND [37]). Let $\left(X_{t}\right)_{t}$ be a non-decreasing level process (as detailed in Definition 3.2). Let $\chi, \gamma_{i, j} \in[0,1]$ and $p_{i} \in(0,1]$ be such that

- for all $t$, if $X_{t}=i$, the probability that $X_{t+1}=j$ is at most $p_{i} \cdot \gamma_{i, j}$

- $\sum_{j=i+1}^{m} \gamma_{i, j}=1$; and

- for all $j>i$, we have $\gamma_{i, j} \geq \chi \sum_{k=j}^{m} \gamma_{i, k}$.

Then the expected time for $\left(X_{t}\right)_{t}$ to reach the state $m$ is

$$
E[T] \geq \sum_{i=1}^{m-1} \operatorname{Pr}\left[X_{0}=i\right] \chi \sum_{j=i}^{m-1} \frac{1}{p_{j}} .
$$

This result is much stronger than the original lower bound from Fitness Level Method, since now the leaving probabilities of all segments are part of the bound, at least with a fractional impact prescribed by $\chi$. The weakness of the method is that $\chi$ has to be defined globally, the same for all segments $i$.

There is also a corresponding upper bound as follows.

Theorem 3.7 (Fitness Level Method with Viscosity, upper BOUND [37]). Let $\left(X_{t}\right)_{t}$ be a non-decreasing level process (as detailed in Definition 3.2). Let $\chi, \gamma_{i, j} \in[0,1]$ and $p_{i} \in(0,1]$ be such that

- for all $t$, if $X_{t}=i$, the probability that $X_{t+1}=j$ is at least $p_{i} \cdot \gamma_{i, j}$

- $\sum_{j=i+1}^{m} \gamma_{i, j}=1$;

- for all $j>i$, we have $\gamma_{i, j} \leq \chi \sum_{k=j}^{m} \gamma_{i, k}$; and

- for all $j \leq m-2$, we have $(1-\chi) p_{j} \leq p_{j+1}$.
Then the expected time for $\left(X_{t}\right)_{t}$ to reach the state $m$ is

$$
E[T] \leq \sum_{i=1}^{m-1} \operatorname{Pr}\left[X_{0}=i\right]\left(\frac{1}{p_{j}}+\chi \sum_{j=i+1}^{m-1} \frac{1}{p_{j}}\right) .
$$

\subsection{Fitness Level Method with Visit Probabilities}

In this paper, we give a new FLM theorem for proving lower bounds. The idea is that exactly all those levels that have ever been visited need to be left; thus, we can use the expected waiting time for leaving a specific level multiplied with the probability of visiting that level at all. The following theorem makes this idea precise for lower bounds; Theorem 3.9 gives the corresponding upper bound. We note that for the particular case of the optimization of the LEADINGONEs problem via (1+1)-type elitist algorithms, our bounds are special cases of [12, Lemma 5] and [6, Theorem 3].

Theorem 3.8 (Fitness Level Method with visit probabiliTIES, LOWER BOUND). Let $\left(X_{t}\right)_{t}$ be a non-decreasing level process (as detailed in Definition 3.2). For all $i \in[1 . . m-1]$, let $p_{i}$ be an upper bound on the probability of a state change of $\left(X_{t}\right)_{t}$, conditional on being in state i. Furthermore, let $v_{i}$ be a lower bound on the probability of there being a $t$ such that $X_{t}=i$. Then the expected time for $\left(X_{t}\right)_{t}$ to reach the state $m$ is

$$
E[T] \geq \sum_{i=1}^{m-1} \frac{v_{i}}{p_{i}}
$$

Proof. For each $i<m$, let $T_{i}$ be the (random) time spent in level $i$. Thus,

$$
T=\sum_{i=1}^{m-1} T_{i}
$$

Let now $i<m$. We want to show that $E\left[T_{i}\right] \geq v_{i} / p_{i}$. We let $E$ be the event that the process ever visits level $i$ and compute

$$
E\left[T_{i}\right]=E\left[T_{i} \mid E\right] \operatorname{Pr}[E]+E\left[T_{i} \mid \bar{E}\right] \operatorname{Pr}[\bar{E}] \geq E\left[T_{i} \mid E\right] v_{i} .
$$

For all $t$ with $X_{t}=i$, with probability at most $p_{i}$, we have $X_{t+1}>i$. Thus, using Lemma 3.3, the expected time until a search point with $X_{k}>i$ is found is at least $1 / p_{i}$, giving $E\left[T_{i} \mid E\right] \geq 1 / p_{i}$ as desired.

A strength of this formulation is that skipping levels due to a higher initialization does not need to be taken into account separately (as in the two previous lower bounds), it is part of the visit probabilities. A corresponding upper bound follows with analogous arguments.

Theorem 3.9 (Fitness Level Method With Visit ProbabiliTIES, UPPER BOUND). Let $\left(X_{t}\right)_{t}$ be a non-decreasing level process (as detailed in Definition 3.2).

For all $i \in[1 . . m-1]$, let $p_{i}$ be a lower bound on the probability of a state change of $\left(X_{t}\right)_{t}$, conditional on being in state $i$. Furthermore, let $v_{i}$ be an upper bound on the probability there being a $t$ such that $X_{t}=i$. Then the expected time for $\left(X_{t}\right)_{t}$ to reach the state $m$ is

$$
E[T] \leq \sum_{i=1}^{m-1} \frac{v_{i}}{p_{i}}
$$


In a typical application of the method of the FLM, finding good estimates for the leaving probabilities is easy. It is more complicated to estimate the visit probabilities accurately, so we propose one possible approach in the following lemma.

LEMma 3.10. Let $\left(Y_{t}\right)_{t}$ be a Markov-process over state space $S$ and $\ell: S \rightarrow[1 . . m]$ a level function. For all $t$, let $X_{t}=\ell\left(Y_{t}\right)$ and suppose that $\left(X_{t}\right)_{t}$ is non-decreasing. Further, suppose that $\left(X_{t}\right)_{t}$ reaches state $m$ after a finite time with probability 1 .

Let $i<m$ be given. For any $x \in S$ and any set $M \subseteq S$, let $x \rightarrow M$ denote the event that the Markov chain with current state $x$ transitions to a state in $M$. For all $j$ let $A_{j}=\{s \in S \mid \ell(s)=j\}$. Suppose there is $v_{i}$ such that, for all $x \in A_{\leq i-1}$ with $\operatorname{Pr}\left[x \rightarrow A_{\geq i}\right]>0$,

$$
\operatorname{Pr}\left[x \rightarrow A_{i} \mid x \rightarrow A_{\geq i}\right] \geq v_{i},
$$

and

$$
\operatorname{Pr}\left[Y_{0} \in A_{i} \mid Y_{0} \in A_{\geq i}\right] \geq v_{i} .
$$

Then $v_{i}$ is a lower bound for visiting level $i$ as required by Theorem 3.8.

Proof. Let $T$ be minimal such that $Y_{T} \in A_{\geq i}$. Then the probability that level $i$ is being visited is $\operatorname{Pr}\left[Y_{T} \in A_{i}\right]$, since $\left(X_{t}\right)_{t}$ is non-decreasing.

By the law of total probability we can show the claim by showing it first conditional on $T=0$ and then conditional on $T \neq 0$.

We have that $T=0$ is equivalent to $Y_{0} \in A_{\geq i}$, thus we have $\operatorname{Pr}\left[Y_{T} \in A_{i} \mid T=0\right] \geq v_{i}$ from the second condition in the statement of the lemma.

Otherwise, let $x=Y_{T-1}$. Since $Y_{T} \in A_{\geq i}$,

$$
\begin{aligned}
\operatorname{Pr}\left[Y_{T} \in A_{i} \mid T \neq 0\right] & =\operatorname{Pr}\left[Y_{T} \in A_{i} \mid Y_{T} \in A_{\geq i}, T \neq 0\right] \\
& =\operatorname{Pr}\left[x \rightarrow A_{i} \mid x \rightarrow A_{\geq i}, T \neq 0\right] \\
& =\operatorname{Pr}\left[x \rightarrow A_{i} \mid x \rightarrow A_{\geq i}\right] .
\end{aligned}
$$

As $T$ was chosen minimally, we have $x \notin A_{\geq i}$ and thus get the desired bound from the first condition in the statement of the lemma.

Implicitly, the lemma suggests to take the minimum of all these conditional probabilities over the different choices for $x$. Note that this estimate might be somewhat imprecise since worst-case $x$ might not be encountered frequently. Also note that a corresponding upper bound for Theorem 3.9 follows analogously.

\section{THE PRECISE RUN TIME FOR LEADINGONES}

One of the classic fitness functions used for analyzing the optimization behavior of randomized search heuristics is the LEADINGONES function. Given a bit string $x$ of length $n$, the LEADINGOnES value of $x$ is defined as the number of $1 \mathrm{~s}$ in the bit string before the first 0 (if any). In parallel independent work, the precise expected run time of the $(1+1)$ EA on the LEADINGONEs benchmark function was determined in $[2,37]$. Even more, the distribution of the run time was determined with variants of the FLM in $[6,12]$. As a first simple application of our methods, we now determine the precise run time of the $(1+1)$ EA on LEAdINGOnES via Theorems 3.8 and 3.9.

Theorem 4.1. Consider the $(1+1)$ EA optimizing LEADINGONES with mutation rate p. Let $T$ be the (random) time for the $(1+1) E A$ to find the optimum. Then

$$
E[T]=\frac{1}{2} \sum_{i=0}^{n-1} \frac{1}{(1-p)^{i} p} .
$$

Proof. We want to apply Theorems 3.8 and 3.9 simultaneously. We partition the search space in the canonical way such that, for all $i \leq n, A_{i}$ contains the set of all search points with fitness $i$. Now we need a precise result for the probability to leave a level and for the probability to visit a level.

First, we consider the probability $p_{i}$ to leave a given level $i<n$. Suppose the algorithm has a current search point in $A_{i}$, so it has $i$ leading $1 \mathrm{~s}$ and then a 0 . The algorithm leaves level $A_{i}$ now if and only if it flips the first 0 of the bit string (probability of $p$ ) and no previous bits (probability $\left.(1-p)^{i}\right)$. Hence, $p_{i}=p(1-p)^{i}$.

Next we consider the probability $v_{i}$ to visit a level $i$. We claim that it is exactly $1 / 2$, following reasoning given in several places before $[18,37]$. We want to use Lemma 3.10 and its analog for upper bounds. Let $i$ be given. For the initial search point, if it is at least on level $i$ (the condition considered by the lemma), the individual is on level $i$ if and only if the $i+1$ st bit is a 0 , so exactly with probability $1 / 2$ as desired for both bounds. Before an individual with at least $i$ leading $1 \mathrm{~s}$ is created, the bit at position $i+1$ remains uniformly random (this can be seen by induction: it is uniform at the beginning and does not experience any bias in any iteration while no individual with at least $i$ leading 1 is created). Once such an individual is created, if the bit at position $i+1$ is 1 , the level $i$ is skipped, otherwise it is visited. Thus, the algorithm skips level $i$ with probability exactly $1 / 2$, giving $v_{i}=1 / 2$. With these exact values for the $p_{i}$ and $v_{i}$, Theorems 3.8 and 3.9 immediately yield the claim.

By computing the geometric series in Theorem 4.1, we obtain as a (well-known) corollary that the $(1+1)$ EA with the classic mutation rate $p=1 / n$ optimizes LEADINGONES in an expected run time of $n^{2} \frac{e-1}{2}(1 \pm o(1))$.

\section{A TIGHT LOWER BOUND FOR ONEMAX}

In this section, as a first real example of the usefulness of our general method, we prove a lower bound for the run time of the $(1+1)$ EA with standard mutation rate $p=\frac{1}{n}$ on ONEMAX, which is only by an additive term of order $O(n)$ below the upper bound following from the classic fitness level method. This is tighter than the best gap of order $O(n \log \log n)$ proven previously with fitness level arguments. Moreover, our lower bound is the tightest lower bound apart from the significantly more complicated works that determine the run time precise apart from $o(n)$ terms. We defer a detailed account of the literature together with a comparison of the methods to Section 5.3.

We recall that the fitness levels of the ONEMAx function are given by

$$
A_{i}:=\left\{x \in\{0,1\}^{n} \mid \mathrm{OM}(x)=i\right\}, i \in[0 . . n] .
$$

We use the notation $A_{\geq i}:=\bigcup_{j=i}^{n} A_{j}$ and $A_{\leq i}:=\bigcup_{j=0}^{i} A_{j}$ for all $i \in[0 . . n]$ as defined above for fitness-based partitions, but with the appropriate bounds 0 and $n$ instead of 1 and $m$.

We denote by $T_{k, \ell}$ the expected number of iterations the $(1+1)$ EA, started with a search point in $A_{k}$, takes to generate 
a search point in $A_{\geq \ell}$. We further denote by $T_{\text {rand, } \ell}$ the expected number of iterations the $(1+1)$ EA started with a random search point takes to generate a solution in $A_{\geq \ell}$. These notions extend previously proposed fine-grained run times notions: $T_{\text {rand }, \ell}$ is the fixed target run time first proposed in [12] as a technical tool and advocated more broadly in [3]. The time $T_{k, n}$ until the optimum is found when starting with fitness $k$ was investigated in [1] when $k>n / 2$, that is, when starting with a better-than-average solution. We spare the details and only note that such fine-grained complexity notions (which also include the fixed-budget complexity proposed in [26]) have given a much better picture on how to use EAs effectively than the classic run time $T_{\text {rand, } n}$ alone. In particular, it was observed that different parameters or algorithms are preferable when not optimizing until the optimum or when starting with a good solution.

For all $k, \ell \in[0 . . n]$, we denote by $p_{k, \ell}$ the probability that standard bit mutation with mutation rate $p=\frac{1}{n}$ creates an offspring in $A_{\ell}$ from a parent in $A_{k}$. We also write $p_{k, \geq \ell}:=\sum_{j=\ell}^{n} p_{k, j}$ to denote the probability to generate an individual in $A_{\geq \ell}$ from a parent in $A_{k}$. Then $p_{i}:=p_{i, \geq i+1}$ is the probability that the $(1+1)$ EA optimizing OnEMAX leaves the $i$-th fitness level.

\subsection{Upper and Lower Bounds Via Fitness Levels}

Using the notation just introduced, the classic fitness level method (see Theorem 3.4 and note that the fitness of the parent individuals describes a non-decreasing level process with state change probabilities $p_{i}$ ) shows that

$$
T_{k, \ell} \leq \sum_{i=k}^{\ell-1} \frac{1}{p_{i}}=: \tilde{T}_{k, \ell} .
$$

To prove a nearly matching lower bound employing our new methods, we first analyze the probability that the $(1+1)$ EA optimizing ONEMAx skips a particular fitness level. Note that if $q_{i}$ is the probability to skip the $i$-th fitness level, then $v_{i}:=1-q_{i}$ is the probability to visit the $i$-th level as used in Theorem 3.8.

Lemma 5.1. Let $i \in[0 . . n]$. Consider a run of the $(1+1) E A$ with mutation rate $p=\frac{1}{n}$ on the ONEMAX function started with a (possibly random) individual $x$ with $\operatorname{ONEMAX}_{(x)}<i$. Then the probability $q_{i}$ that during the run the parent individual never has fitness $i$ satisfies

$$
q_{i} \leq \frac{n-i}{n\left(1-\frac{1}{n}\right)^{i-1}} .
$$

Proof. Since we assume that we start below fitness level $i$, by Lemma 3.10 (and using the notation from that lemma for a moment) we have

$$
\begin{aligned}
q_{i} & \leq \max \left\{\operatorname{Pr}\left[x \rightarrow A_{\geq i+1} \mid x \rightarrow A_{\geq i}\right] \mid \operatorname{OnEMAx}(x)<i\right\} \\
& \leq \max _{k \in[0 . . i-1]} \frac{p_{k, \geq i+1}}{p_{k, \geq i}} .
\end{aligned}
$$

Hence it suffices to show that $\frac{p_{k, \geq i+1}}{p_{k, \geq i}} \leq \frac{n-i}{n\left(1-\frac{1}{n}\right)^{i-1}}$ for all $k \in[0 . . i-$ $1]$, and this is what we will do in the remainder of this proof.

Let us, slightly abusing the common notation, write $\operatorname{Bin}(m, p)$ to denote a random variable following a binomial law with parameters $m$ and $p$. Let $k, \ell \in \mathbb{N}$ with $k \leq \ell$. Noting that the only way to generate a search point in $A_{\ell}$ from some $x \in A_{k}$ is to flip, for some $j \in[\ell-k . . \min \{n-k, \ell\}]$, exactly $j$ of the $n-k$ zero-bits of $x$ and exactly $j-(\ell-k)$ of the $k$ one-bits, we easily obtain the well-known fact that

$$
\begin{aligned}
p_{k, \ell} & =\sum_{j=\ell-k}^{\min \{n-k, \ell\}} \operatorname{Pr}[\operatorname{Bin}(n-k, p)=j] \operatorname{Pr}[\operatorname{Bin}(k, p)=j-(\ell-k)] \\
& =\sum_{j=\ell-k}^{\min \{n-k, \ell\}}\left(\begin{array}{c}
n-k \\
j
\end{array}\right)\left(\begin{array}{c}
k \\
j-(\ell-k)
\end{array}\right) p^{2 j-\ell+k}(1-p)^{n-2 j+\ell-k} .
\end{aligned}
$$

Since $p=\frac{1}{n}$, the mode of $\operatorname{Bin}(n-k, p)$ is at most 1 . Since the binomial distribution is unimodal, we conclude that $\operatorname{Pr}[\operatorname{Bin}(n-k, p)=j] \leq$ $\operatorname{Pr}[\operatorname{Bin}(n-k, p)=\ell-k]$ for all $j \geq \ell-k$. Consequently, the first line of the above set of equations gives

$$
\begin{aligned}
p_{k, \ell} & \leq \operatorname{Pr}[\operatorname{Bin}(n-k, p)=\ell-k] \operatorname{Pr}[\operatorname{Bin}(k, p) \in[0 . . \min \{n-\ell, k\}]] \\
& \leq \operatorname{Pr}[\operatorname{Bin}(n-k, p)=\ell-k]
\end{aligned}
$$

and thus

$$
p_{k, \geq \ell} \leq \operatorname{Pr}[\operatorname{Bin}(n-k, p) \geq \ell-k] .
$$

We recall that our target is to estimate $\frac{p_{k, \geq i+1}}{p_{k, \geq i}}$ for all $k \in[0 . . i-1]$. By (1), we have

$$
\begin{aligned}
p_{k, \geq i+1} & \leq \operatorname{Pr}[\operatorname{Bin}(n-k, p) \geq i+1-k] \\
& \leq \frac{(i+1-k)(1-p)}{i+1-k-(n-k) p} \operatorname{Pr}[\operatorname{Bin}(n-k, p)=i+1-k],
\end{aligned}
$$

where the last estimate is [7, equation following Lemma 1.10.38]. We also have $p_{k, \geq i} \geq p_{k, i} \geq(1-p)^{k} \operatorname{Pr}[\operatorname{Bin}(n-k, p)=i-k]$. Hence from

$$
\begin{aligned}
\frac{\operatorname{Pr}[\operatorname{Bin}(n-k, p)=i+1-k]}{\operatorname{Pr}[\operatorname{Bin}(n-k, p)=i-k]} & =\frac{\left(\begin{array}{c}
n-k \\
i+1-k
\end{array}\right) p^{i+1-k}(1-p)^{n-k-(i+1-k)}}{\left(\begin{array}{c}
n-k \\
i-k
\end{array}\right) p^{i-k}(1-p)^{n-k-(i-k)}} \\
& =\frac{(n-i) p}{(i+1-k)(1-p)}
\end{aligned}
$$

we conclude

$$
\begin{aligned}
\frac{p_{k, \geq i+1}}{p_{k, \geq i}} & \leq \frac{(i+1-k)(1-p)}{i+1-k-(n-k) p} \frac{(n-i) p}{(i+1-k)(1-p)^{k+1}} \\
& \leq \frac{n-i}{n(i-k)\left(1-\frac{1}{n}\right)^{k}},
\end{aligned}
$$

using again that $p=\frac{1}{n}$. For $k \in[0 . . i-1]$, this expression is maximal for $k=i-1$, giving that $q_{i} \leq \frac{n-i}{n\left(1-\frac{1}{n}\right)^{i-1}}$ as claimed.

With this estimate, we can now easily give a very tight lower bound on the run time of the $(1+1)$ EA on ONEMAX.

Theorem 5.2. Let $k, \ell \in[0 . . n]$ with $k<\ell$. Then the expected number $T_{k, \ell}$ of iterations the $(1+1)$ EA optimizing ONEMAX and initialized with any search point $x$ with $\operatorname{ONEMAX}(x)=k$ takes to generate a search point $z$ with fitness $\operatorname{ONEMAX}(z) \geq \ell$ is at least

$$
T_{k, \ell} \geq \tilde{T}_{k, \ell}-(\ell-k-1) e(e-1) \exp \left(\frac{k}{n-1}\right),
$$

where $\tilde{T}_{k, \ell}$ is the upper bound stemming from the fitness level method as defined at the beginning of this section. This lower bound holds also for $T_{k^{\prime}, \ell}$ with $k^{\prime} \leq k$, that is, when starting with a search point $x$ with $\operatorname{ONEMAX}(x) \leq k$. 
Proof. We use our main result Theorem 3.8. We note first that when assuming that the level process regarded in Theorem 3.8 starts on level $k^{\prime}$, then the expected time for it to reach level $\ell$ or higher is at least $\sum_{i=k^{\prime}}^{\ell-1} \frac{v_{i}}{p_{i}}$. This follows immediately from the proof of the theorem or by applying the theorem to the level process $\left(X_{t}^{\prime}\right)$ defined by $X_{t}^{\prime}=\min \left\{\ell, X_{t}\right\}-k^{\prime}$ for all $t$.

Consider now a run of the $(1+1)$ EA on the ONEMAx function started with an initial search point $x_{0}$ such that $k^{\prime}=$ $\operatorname{OneMax}\left(x_{0}\right) \leq k$. Denote by $x_{t}$ the individual selected in iteration $t$ as future parent. Then $X_{t}=\operatorname{OnEMax}\left(x_{t}\right)$ defines a level process. As before, we denote the probabilities to visit level $i$ by $v_{i}$, to not visit it by $q_{i}=1-v_{i}$, and to leave it to a higher level by $p_{i}$. Using our main result and the elementary argument above, we obtain an expected run time of

$$
E\left[T_{k^{\prime}, \ell}\right] \geq \sum_{i=k^{\prime}}^{\ell-1} \frac{v_{i}}{p_{i}} \geq \sum_{i=k}^{\ell-1} \frac{v_{i}}{p_{i}} \geq \sum_{i=k}^{\ell-1} \frac{1}{p_{i}}-\sum_{i=k+1}^{\ell-1} \frac{q_{i}}{p_{i}} .
$$

We note that the first expression is exactly the upper bound $\tilde{T}_{k, \ell}$ stemming from the classic fitness level method. We estimate the second expression. We have

$$
p_{i}=p_{i, \geq i+1} \geq p_{i, i+1} \geq\left(1-\frac{1}{n}\right)^{n-1} \frac{n-i}{n},
$$

where the last estimate stems from regarding only the event that exactly one missing bit is flipped. Together with the estimate $q_{i} \leq$ $\frac{n-i}{n\left(1-\frac{1}{n}\right)^{i-1}}$ from Lemma 5.1, we compute

$$
\begin{aligned}
\sum_{i=k+1}^{\ell-1} \frac{q_{i}}{p_{i}} & \leq \sum_{i=k+1}^{\ell-1} \frac{n-i}{n\left(1-\frac{1}{n}\right)^{i-1}} \frac{n}{(n-i)\left(1-\frac{1}{n}\right)^{n-1}} \\
& =\sum_{i=k+1}^{\ell-1}\left(1+\frac{1}{n-1}\right)^{n+i-2} \\
& =\left(1+\frac{1}{n-1}\right)^{n+k-1} \sum_{j=0}^{\ell-k-2}\left(1+\frac{1}{n-1}\right)^{j} \\
& =\left(1+\frac{1}{n-1}\right)^{n+k-1} \frac{\left(1+\frac{1}{n-1}\right)^{\ell-k-1}-1}{\left(1+\frac{1}{n-1}\right)-1} \\
& =\left(1+\frac{1}{n-1}\right)^{n+k-1}(n-1)\left(\left(1+\frac{1}{n-1}\right)^{\ell-k-1}-1\right) \\
& \leq(n-1) \exp \left(\frac{n+k-1}{n-1}\right)\left(\exp \left(\frac{\ell-k-1}{n-1}\right)-1\right) \\
& =(n-1) e \exp \left(\frac{k}{n-1}\right)\left(\exp \left(\frac{\ell-k-1}{n-1}\right)-1\right) \\
& \leq(n-1)(e-1) e \exp \left(\frac{k}{n-1}\right) \frac{\ell-k-1}{n-1},
\end{aligned}
$$

where the estimate in (3) uses the well-known inequality $1+r \leq e^{r}$ valid for all $r \in \mathbb{R}$ and the last estimate exploits the convexity of the exponential function in the interval $[0,1]$, that is, that $\exp (\alpha) \leq$ $1+\alpha(\exp (1)-\exp (0))$ for all $\alpha \in[0,1]$.

The result above shows that the classic fitness level method and our new lower bound method can give very tight run time results. We note that the difference $\delta_{k, \ell}=(\ell-k-1) e(e-1) \exp \left(\frac{k}{n-1}\right)$ between the two fitness level estimates is only of order $O(\ell-k)$, in particular, only of order $O(n)$ for the classic run time $T_{\text {rand, } n}$, which itself is of order $\Theta(n \log n)$. Hence here the gap is only a term of lower order.

\subsection{Estimating the Fitness Level Estimate $\tilde{T}_{k, \ell}$}

To make our results above meaningful, it remains to analyze the quantity $\tilde{T}_{k, \ell}=\sum_{i=k}^{\ell-1} 1 / p_{i}$, which is the estimate from the classic fitness level method. Due to space constraints, some proofs had to be omitted in this section. They can be found in the extended version [15].

TheOREM 5.3. The expected number of iterations the $(1+1) E A$ optimizing ONEMAX, started with a search point of fitness $k$, takes to find a search point with fitness $\ell$ or larger, satisfies

$$
\begin{aligned}
& e_{n} n \sum_{i=n-\ell+1}^{n-k} \frac{1}{i}-(\ell-k-1) e(e-1) \exp \left(\frac{k}{n-1}\right)-\frac{1}{2} e_{n}^{2}(\ell-k) \\
& \leq T_{k, \ell} \leq e_{n} n \sum_{i=n-\ell+1}^{n-k} \frac{1}{i}, \\
& \text { where } e_{n}:=\left(1-\frac{1}{n}\right)^{-(n-1)} .
\end{aligned}
$$

When starting the $(1+1)$ EA with a random initial search point, the following bounds apply.

TheOREM 5.4. There is an absolute constant $K$ such that the expected run time $T=T_{\text {rand, } n}$ of the $(1+1) E A$ with random initialization on ONEMAX satisfies

$$
e_{n} n \sum_{i=1}^{\lceil n / 2\rceil} \frac{1}{i}-4.755 n-K \leq T \leq e_{n} n \sum_{i=1}^{\lceil n / 2\rceil} \frac{1}{i}+K .
$$

In particular,

$$
\text { en } \ln (n)-4.871 n-O(\log n) \leq T \leq e n \ln (n)-0.115 n+O(1) .
$$

\subsection{Comparison with the Literature}

We end this section by giving an overview on the previous works analyzing the run time of the $(1+1)$ EA on OnEMAX and comparing them to our result. Some of the results described in the following, in particular, Sudholt's lower bound [37], were also proven for general mutation rates $p$ instead of only $p=\frac{1}{n}$. To ease the comparison with our result, we only state the results for the case that $p=\frac{1}{n}$. We note that with our method we could also have analysed broader ranges of mutation rates. The resulting computations, however, would have been more complicated and would have obscured the basic application of our method.

To the best of our knowledge, the first to state and rigorously prove a run time bound for this problem was Rudolph in his dissertation [34, p. 95], who showed that $T=T_{\text {rand, } n}$ satisfies $E[T] \leq\left(1-\frac{1}{n}\right)^{n-1} n \sum_{i=1}^{n} \frac{1}{i}$, which is exactly the upper bound $\tilde{T}_{0, n}^{+}$from the fitness level method and from only regarding the events that levels are left via one-bit flips. A lower bound of $n \ln (n)-O(n \log \log n)$ was shown in [17] for the optimization of a general separable function with positive weights when starting in the search point $(0, \ldots, 0)$. From the proof of this result, it is clear that it holds for any pseudo-Boolean function with unique global 
optimum $(1, \ldots, 1)$. This lower bound builds on the argument that each bit needs to be flipped at least once in some mutation step. It is not difficult to see that the expected time until this event happens is indeed $(1 \pm o(1)) n \ln n$, so this argument is too weak to make the leading constant of $E[T]$ precise.

Only a very short time after these results and thus quite early in the young history of run time analysis of evolutionary algorithms, Garnier, Kallel, and Schoenauer [20] showed that $E[T]=$ $e n \ln (n)+c_{1} n+o(n)$ for a constant $c_{1} \approx-1.9$, however, the completeness of their proof has been doubted in [23]. Since at that early time precise run time analyses were not very popular, it took a while until Doerr, Fouz, and Witt [10] revisited this problem and showed with $E[T] \geq(1-o(1)) e n \ln (n)$ the first lower bound that made the leading constant precise. Their proof used a variant of additive drift from [25] together with the potential function $\ln \left(Z_{t}\right)$, where $Z_{t}$ denotes the number of zeroes in the parent individual at time $t$. Shortly later, Sudholt [36] (journal version [37]) used his fitness level method for lower bounds to show $E[T] \geq e n \ln (n)-2 n \log \log n-16 n$. That the run time was $E[T]=e n \ln (n)-\Theta(n)$ was proven first in [11], where an upper bound of en $\ln (n)-0.1369 n+O(1)^{1}$ was shown via variable drift for upper bounds [27, 32] and a lower bound of $E[T] \geq$ en $\ln (n)-O(n)$ was shown via a new variable drift theorem for lower bounds on hitting times. An explicit version of the lower bound of en $\ln (n)-7.81791 n-O(\log n)$ and an alternative proof of the upper bound en $\ln (n)-0.1369 n+O(1)$ was given in [30] via a very general drift theorem.

The final answer to this problem was given in an incredibly difficult work by Hwang, Panholzer, Rolin, Tsai, and Chen [23] (see [24] for a simplified version), who showed

$$
E[T]=e n \ln (n)+c_{1} n+\frac{1}{2} e \ln (n)+c_{2}+O\left(n^{-1} \log n\right)
$$

with explicit constants $c_{1} \approx-1.9$ and $c_{2} \approx 0.6$.

In the light of these results, we feel that our proof of an en $\ln (n) \pm$ $O(n)$ bound is the first simple proof a run time estimate of this precision for this problem. Interestingly, our explicit lower bound en $\ln (n)-4.871 n-O(\log n)$ is even a little stronger than the bound en $\ln (n)-7.81791 n-O(\log n)$ proven with drift methods in [30].

\section{A BOUND FOR LONG $k$-PATHS}

Long $k$-paths, introduced in [33], have been studied in various places; we point the reader to [35] for a discussion, which also contains the formalization that we use. A lower bound for long $k$-paths using FLM with viscosities was given in [37].

We use [35, Lemma 3] (phrased as a definition below) and need to know no further details about what a long $k$-path is. In fact, our proof uses all the ideas of the proof of [37], but cast in terms of our FLM with visit probabilities, which, we believe, makes the proof simpler and the core ideas more prominent. Note that [37] first needs to extend the FLM with viscosities by introducing an additional parameter before it is applicable in this case.

Definition 6.1. Let $k, n$ be given such that $k$ divides $n$. A long $k$-path is function $f:\{0,1\}^{n} \rightarrow \mathbb{R}$ with the following properties.

- The 0-bit string has a fitness of 0 ; there are $m=k 2^{n / k}-k$ bit strings of positive fitness, and all these values are distinct;

\footnotetext{
${ }^{1}$ The constant 0.1369 was wrongly stated as 0.369 as pointed out in [30]
}

all other bit strings have negative fitness. We call the bit strings with non-negative fitness as being on the path and consider them ordered by fitness (this way we can talk about the "next" element on the path and similar).

- For each bit string with non-negative fitness and each $i<k$, the bit string with $i$-next higher fitness is exactly a Hamming distance of $i$ away.

- For each bit string with non-negative fitness and each $i \geq k$, the bit string with $i$-next higher fitness is at least a Hamming distance of $k$ away.

For an explicit construction of a long $k$-path, see [18, 35]. The long $k$-paths are designed such that optimization proceeds by following the (long) path and true shortcuts are unlikely, since they require jumping at least $k$.

The following lower bound for optimizing long $k$-paths with the $(1+1)$ EA is given in [37]. Note that $n$ is the length of the bit strings, $m$ is the length of the path and $p$ is the mutation rate.

$$
m \frac{1-2 p}{p(1-p)^{n}} \frac{1-2 p}{1-p}\left(1-\left(\frac{p}{1-p}\right)^{k}\right)^{m} \text {. }
$$

We want to show here that we can derive the essentially same bound with the same ideas but less technical details.

Note that the lower bound given in [37] is only meaningful for $k \geq \sqrt{n / \log (1 / p)}$, as the last term of the bound would otherwise be close to 0 :

$$
\begin{aligned}
\left(1-\left(\frac{p}{1-p}\right)^{k}\right)^{m} & \leq\left(1-p^{k}\right)^{m} \leq \exp \left(-m p^{k}\right) \\
& \leq \exp \left(-2^{n / k} p^{k}\right)=\exp \left(-2^{n / k-k \log (1 / p)}\right)
\end{aligned}
$$

We have that $n / k-k \log (1 / p)$ is positive if and only if $n / \log (1 / p) \geq$ $k^{2}$. In fact, if $k=\omega(\sqrt{n / \log (1 / p)})$, we have

$$
\begin{aligned}
\left(1-\left(\frac{p}{1-p}\right)^{k}\right)^{m} & \geq\left(1-(2 p)^{k}\right)^{m} \geq 1-m(2 p)^{k} \\
& \geq 1-2^{3 n / k-k \log (1 / p)} \\
& =1-2^{\sqrt{n} o(\log (1 / p))-\sqrt{n} \omega(\log (1 / p))} \\
& =1-2^{-\sqrt{n} \omega(\log (1 / p))} \geq 1-2^{-\sqrt{n}} .
\end{aligned}
$$

This also entails $p \leq \exp \left(-n / k^{2}\right)$.

With our fitness level method, we obtain the following lower bound. It differs from Sudholt's bound (4) by an additional term $m$, which reduces the lower bound. Analyzing why this term does not appear in Sudholt's analysis, we note that the $\gamma_{i, j}$ chosen in [37] are underestimating the true probability to jump to elements of the path that are more than $k$ steps (on the path) away. When this is corrected, as confirmed to us by the author, Sudholt's proof would also only show our bound below.

Theorem 6.2. Consider the $(1+1)$ EA on a long $k$-path of length $m$ with mutation rate $p \leq 1 / 2$ starting at the all-0 bit string (the start of the path). ${ }^{2}$ Let $T$ be the (random) time for the $(1+1)$ EA to find

\footnotetext{
${ }^{2}$ This simplifying assumption about the start point was also made in [37].
} 
the optimum. Then

$$
E[T] \geq m \frac{1-2 p}{p(1-p)^{n}} \frac{1-2 p}{1-p}\left(1-m\left(\frac{p}{1-p}\right)^{k-1}\right)^{m} .
$$

Proof. We are setting up to apply Theorem 3.8. We partition the search space in the canonical way such that, for all $i \leq m$ with $i>0, A_{i}$ contains the only $i$-th point of the path and nothing else, and $A_{0}$ contains all points not on the path. In order to simplify the analysis, we will first change the behavior of the algorithm such that it discards any offspring which differs from its parent by at least $k$ bits. This will allow us to apply Theorem 3.8 quickly and cleanly, afterwards we will show that the progress of this modified algorithm is very close to the progress of the original algorithm.

In this modified process, we first consider the probability $p_{i}$ to leave a given level $i<m$. For this, the algorithm has to jump up exactly $j<k$ fitness levels, which is achieved by flipping a specific set of $j$ bits; the probability for this is

$$
\begin{aligned}
p_{i} & =\sum_{j=1}^{k-1} p^{j}(1-p)^{n-j} \leq(1-p)^{n} \sum_{j=1}^{\infty}\left(\frac{p}{1-p}\right)^{j} \\
& =(1-p)^{n} \frac{p /(1-p)}{1-p /(1-p)}=p(1-p)^{n} \frac{1}{1-2 p} .
\end{aligned}
$$

Next we consider the probability $v_{i}$ to visit a level $i$. We want to apply Lemma 3.10, so let some $x \in A_{<i}$ be given, on level $\ell(x)$. Let $d=i-\ell(x)$. Note that $d$ is the Hamming distance between $x$ and the unique point in $A_{i}$. Thus, in case of $d \geq k$, we have $\operatorname{Pr}\left[x \rightarrow A_{i}\right]=0$, so suppose $d<k$. Then we have

$$
\begin{gathered}
\operatorname{Pr}\left[x \rightarrow A_{i} \mid x \rightarrow \bigcup_{j=i}^{m} A_{j}\right]=\frac{\operatorname{Pr}\left[x \rightarrow A_{i}\right]}{\operatorname{Pr}\left[x \rightarrow \bigcup_{j=i}^{m} A_{j}\right]} \\
=\frac{p^{d}(1-p)^{n-d}}{\sum_{j=d}^{k} p^{j}(1-p)^{n-j}}=\frac{1-2 p}{1-p} .
\end{gathered}
$$

By Lemma 3.10, we can use this last term as $v_{i}$ in Theorem 3.8 (it also fulfills the second condition of Lemma 3.10, since the process starts deterministically in the 0 string). Note that neither $p_{i}$ nor $v_{i}$ depends on $i$. Using Theorem 3.8 and recalling that we have $m$ levels, we get a lower bound of

$$
m \frac{1-2 p}{p(1-p)^{n}} \frac{1-2 p}{1-p}
$$

Note that this is exactly the term derived in [37] except for a term correcting for the possibility of jumps of more than $k$ bits, which we also still need to correct for.

We now show that this probability of making a successful jump of distance at least $k$ is small. To that end we will show that it is very unlikely to leave a fitness level with a large jump rather than just move to the next level. Suppose the algorithm is currently at $x \in A_{i}$. Leaving $x$ with a jump of at least $k$ to a specific element on the path is less likely the longer the jump is (since $p \leq 1 / 2$ ). Thus, we can upper bound the probability of jumping to an element of the path which is more than $k$ away as $p^{k}(1-p)^{n-k}$. Thus, conditional on leaving the fitness level, the probability of leaving it with a $\geq k$-jump is

$$
\operatorname{Pr}\left[x \rightarrow A_{\geq i+k} \mid x \rightarrow A_{>i}\right]=\frac{\operatorname{Pr}\left[x \rightarrow A_{\geq i+k}\right]}{\operatorname{Pr}\left[x \rightarrow A_{>i}\right]}
$$

$$
\leq \frac{m p^{k}(1-p)^{n-k}}{p(1-p)^{n-1}}=m\left(\frac{p}{1-p}\right)^{k-1} .
$$

Thus, the probability of never making an accepted jump of at least $k$ is bounded from below by the probability to, independently once for each of the $m$ fitness levels, leave the fitness level with a 1-step rather than a jump of at least $k$, which is

$$
\left(1-m\left(\frac{p}{1-p}\right)^{k-1}\right)^{m}
$$

By pessimistically assuming that the process takes a time of 0 in case it ever makes an accepted jump of at least $k$, we can lower-bound the expected time of the original process to reach the optimum as the product of the expected time of the modified process times the probability to never make progress of $k$ or more.

\section{CONCLUSION}

In this work, we proposed a simple and natural way to prove lower bounds via fitness level arguments. The key to our approach is that the true run time can be expressed as the sum of the waiting times to leave a fitness level, weighted with the probability that this level is visited at all. When applying this idea, usually the most difficult part is estimating the probabilities to visit the levels, but as our examples LeAdingOnes, OneMax, and long paths show, this is not overly difficult and clearly easier than setting correctly the viscosity parameters of the previous fitness level method for lower bounds. For this reason, we are optimistic that our method will be an effective way to prove other lower bounds in the future, most easily, of course, for problems where upper bounds were proven via fitness level arguments as well.

Our method makes most sense for elitist evolutionary algorithms even though by regarding the best-so-far individual any evolutionary algorithm gives rise to a non-decreasing level process (at the price that the estimates for the level leaving probabilities become weaker). We are optimistic that our method can be extended to non-elitist algorithms, though. We note that the level visit probability $v_{i}$ for an elitist algorithm is equal to the expected number of separate visits to this level (simply because each level is visited exactly once or never). When defining the $v_{i}$ as the expected number of times the $i$-th level is visited, our upper and lower bounds of Theorems 3.8 and 3.9 remain valid (the proof would use Wald's equation). We did not detail this in our work since our main focus were the elitist examples regarded in [37], but we are optimistic that this direction could be interesting to prove lower bounds also for non-elitist algorithms.

\section{ACKNOWLEDGMENTS}

This work was supported by a public grant as part of the Investissements d'avenir project, reference ANR-11-LABX-0056-LMH, LabEx $\mathrm{LMH}$, and by the Deutsche Forschungsgemeinschaft (DFG), grant FR 2988/17-1. 


\section{REFERENCES}

[1] Denis Antipov, Maxim Buzdalov, and Benjamin Doerr. 2020. First steps towards a runtime analysis when starting with a good solution. In Parallel Problem Solving From Nature, PPSN 2020, Part II. Springer, 560-573.

[2] Süntje Böttcher, Benjamin Doerr, and Frank Neumann. 2010. Optimal fixed and adaptive mutation rates for the LeadingOnes problem. In Parallel Problem Solving from Nature, PPSN 2010. Springer, 1-10.

[3] Maxim Buzdalov, Benjamin Doerr, Carola Doerr, and Dmitry Vinokurov. 2020 Fixed-target runtime analysis. In Genetic and Evolutionary Computation Conference, GECCO 2020. ACM, 1295-1303.

[4] Dogan Corus, Duc-Cuong Dang, Anton V. Eremeev, and Per Kristian Lehre. 2018 Level-based analysis of genetic algorithms and other search processes. IEEE Transactions on Evolutionary Computation 22 (2018), 707-719.

[5] Duc-Cuong Dang and Per Kristian Lehre. 2016. Runtime analysis of non-elitist populations: from classical optimisation to partial information. Algorithmica 75 (2016), 428-461.

[6] Benjamin Doerr. 2019. Analyzing randomized search heuristics via stochastic domination. Theoretical Computer Science 773 (2019), 115-137.

[7] Benjamin Doerr. 2020. Probabilistic tools for the analysis of randomized optimization heuristics. In Theory of Evolutionary Computation: Recent Developments in Discrete Optimization, Benjamin Doerr and Frank Neumann (Eds.). Springer 1-87. Also available at https://arxiv.org/abs/1801.06733.

[8] Benjamin Doerr, Carola Doerr, and Timo Kötzing. 2018. Static and self-adjusting mutation strengths for multi-valued decision variables. Algorithmica 80 (2018), 1732-1768.

[9] Benjamin Doerr, Carola Doerr, and Jing Yang. 2020. Optimal parameter choices via precise black-box analysis. Theoretical Computer Science 801 (2020), 1-34.

[10] Benjamin Doerr, Mahmoud Fouz, and Carsten Witt. 2010. Quasirandom evolutionary algorithms. In Genetic and Evolutionary Computation Conference, GECCO 2010. ACM, 1457-1464.

[11] Benjamin Doerr, Mahmoud Fouz, and Carsten Witt. 2011. Sharp bounds by probability-generating functions and variable drift. In Genetic and Evolutionary Computation Conference, GECCO 2011. ACM, 2083-2090.

[12] Benjamin Doerr, Thomas Jansen, Carsten Witt, and Christine Zarges. 2013. A method to derive fixed budget results from expected optimisation times. In Genetic and Evolutionary Computation Conference, GECCO 2013. ACM, 1581-1588.

[13] Benjamin Doerr, Daniel Johannsen, and Carola Winzen. 2012. Multiplicative drift analysis. Algorithmica 64 (2012), 673-697.

[14] Benjamin Doerr and Timo Kötzing. 2019. Multiplicative up-drift. In Genetic and Evolutionary Computation Conference, GECCO 2019. ACM, 1470-1478.

[15] Benjamin Doerr and Timo Kötzing. 2021. Lower Bounds from Fitness Levels Made Easy. CoRR abs/2104.03372 (2021). arXiv:2104.03372

[16] Benjamin Doerr, Timo Kötzing, J. A. Gregor Lagodzinski, and Johannes Lengler. 2020. The impact of lexicographic parsimony pressure for ORDER/MAJORITY on the run time. Theoretical Computer Science 816 (2020), 144-168.

[17] Stefan Droste, Thomas Jansen, and Ingo Wegener. 1998. A Rigorous complexity analysis of the $(1+1)$ evolutionary algorithm for separable functions with boolean inputs. Evolutionary Computation 6 (1998), 185-196.

[18] Stefan Droste, Thomas Jansen, and Ingo Wegener. 2002. On the analysis of the (1+1) evolutionary algorithm. Theoretical Computer Science 276 (2002), 51-81.

[19] Matthias Feldmann and Timo Kötzing. 2013. Optimizing expected path lengths with ant colony optimization using fitness proportional update. In Foundations of Genetic Algorithms, FOGA 2013. ACM, 65-74.

[20] Josselin Garnier, Leila Kallel, and Marc Schoenauer. 1999. Rigorous hitting times for binary mutations. Evolutionary Computation 7 (1999), 173-203.

[21] Christian Gießen and Carsten Witt. 2018. Optimal mutation rates for the $(1+\lambda)$ EA on OneMax through asymptotically tight drift analysis. Algorithmica 80 (2018), 1710-1731.

[22] Jun He and Xin Yao. 2001. Drift analysis and average time complexity of evolutionary algorithms. Artificial Intelligence 127 (2001), 51-81.

[23] Hsien-Kuei Hwang, Alois Panholzer, Nicolas Rolin, Tsung-Hsi Tsai, and Wei-Mei Chen. 2018. Probabilistic analysis of the (1+1)-evolutionary algorithm. Evolutionary Computation 26 (2018), 299-345.

[24] Hsien-Kuei Hwang and Carsten Witt. 2019. Sharp bounds on the runtime of the $(1+1)$ EA via drift analysis and analytic combinatorial tools. In Foundations of Genetic Algorithms, FOGA 2019. ACM, 1-12.

[25] Jens Jägersküpper. 2007. Algorithmic analysis of a basic evolutionary algorithm for continuous optimization. Theoretical Computer Science 379 (2007), 329-347.

[26] Thomas Jansen and Christine Zarges. 2014. Performance analysis of randomised search heuristics operating with a fixed budget. Theoretical Computer Science 545 (2014), 39-58.

[27] Daniel Johannsen. 2010. Random Combinatorial Structures and Randomized Search Heuristics. Ph.D. Dissertation. Universität des Saarlandes.

[28] Jörg Lässig and Dirk Sudholt. 2014. General upper bounds on the runtime of parallel evolutionary algorithms. Evolutionary Computation 22 (2014), 405-437.

[29] Per Kristian Lehre. 2011. Fitness-levels for non-elitist populations. In Genetic and Evolutionary Computation Conference, GECCO 2011. ACM, 2075-2082.
[30] Per Kristian Lehre and Carsten Witt. 2014. Concentrated hitting times of randomized search heuristics with variable drift. In International Symposium on Algorithms and Computation, ISAAC 2014. Springer, 686-697.

[31] Johannes Lengler. 2020. Drift analysis. In Theory of Evolutionary Computation: Recent Developments in Discrete Optimization, Benjamin Doerr and Frank Neumann (Eds.). Springer, 89-131. Also available at https://arxiv.org/abs/1712.00964.

[32] Boris Mitavskiy, Jonathan E. Rowe, and Chris Cannings. 2009. Theoretical analysis of local search strategies to optimize network communication subject to preserving the total number of links. International fournal on Intelligent Computing and Cybernetics 2 (2009), 243-284.

[33] Günter Rudolph. 1996. How mutation and selection solve long path problems in polynomial expected time. Evolutionary Computation 4 (1996), 195-205.

[34] Günter Rudolph. 1997. Convergence Properties of Evolutionary Algorithms. Verlag Dr. Kovăc.

[35] Dirk Sudholt. 2009. The impact of parametrization in memetic evolutionary algorithms. Theoretical Computer Science 410 (2009), 2511-2528.

[36] Dirk Sudholt. 2010. General lower bounds for the running time of evolutionary algorithms. In Parallel Problem Solving from Nature, PPSN 2010, Part I. Springer, 124-133.

[37] Dirk Sudholt. 2013. A new method for lower bounds on the running time of evolutionary algorithms. IEEE Transactions on Evolutionary Computation 17 (2013), 418-435.

[38] Ingo Wegener. 2001. Theoretical aspects of evolutionary algorithms. In Automata, Languages and Programming, ICALP 2001. Springer, 64-78.

[39] Ingo Wegener. 2002. Methods for the analysis of evolutionary algorithms on pseudo-Boolean functions. In Evolutionary Optimization, R. Sarker, X. Yao, and M. Mohammadian (Eds.). Kluwer, 349-369.

[40] Carsten Witt. 2013. Tight bounds on the optimization time of a randomized search heuristic on linear functions. Combinatorics, Probability \& Computing 22 (2013), 294-318.

[41] Carsten Witt. 2014. Fitness levels with tail bounds for the analysis of randomized search heuristics. Inform. Process. Lett. 114 (2014), 38-41. 